\title{
Transport Properties and Equation of State of Quark-Nuclear Matter
}

\author{
C.O. da Graça \\ Departamento de Física, Universidade Federal de Santa Maria, \\ 97.000-990 Santa Maria, RS - Brazil \\ G. Krein \\ Instituto de Física Teórica, Universidade Estadual Paulista, \\ Rua Pamplona 145, 01405-900 São Paulo, SP - Brazil
}

Received on 30 October, 2002

\begin{abstract}
Transport equations for composite nucleons and deconfined quarks in quark-nuclear matter (QNM) are derived. QNM is a many-body system containing hadrons and deconfined quarks. The starting point is a microscopic quark-meson coupling (QMC) Hamiltonian with a density-dependent quark-quark interaction. An effective quark-hadron Hamiltonian containing canonical hadron and quark field operators is constructed using a mapping procedure. For high densities, the effective Hamiltonian contains interactions that lead to quark deconfinement. Transport equations of the Ueling-Ullenbeck-Vlasov type for quarks and nucleons are obtained using standard many-body techniques with the effective quark-hadron Hamiltonian.
\end{abstract}

\section{Introduction}

The study of the properties of high density hadronic matter is one of the most important problems in contemporary physics. The possibility of creating in laboratory a new state of matter through collisions of heavy nuclei has become feasible in recent years, with widespread opportunities for understanding several aspects of the strong interactions and matter in superdense stars and at the origin of the universe. One of the central questions in this field is the identification of the appropriate degrees of freedom to describe the different phases of hadronic matter. At densities much higher than the nuclear saturation density, a phase of deconfined matter composed of quarks and gluons is expected to occur. The study of the properties of such a state is possible with the methods of perturbative quantum chromodynamics (QCD). On the other hand, the study of matter at densities not much higher than the saturation density of nuclear matter - like the one existing in dense stars - is very complicated. The complication is due to the fact that this phase of matter is characterized by nonperturbative QCD phenomena and the use of tractable models and drastic approximations is presently the only practical way of tackling the problem.

The quark-meson coupling (QMC) model [1] is a very useful model to study the different phases of hadronic matter. It is formulated in terms of quark-gluon degrees of freedom and is devised in such a way to incorporate in an explicit way hadron structure in the nuclear many-body problem. In this model, low-density hadronic matter is described as a system of independent baryons interacting through effective scalar- and vector-meson degrees of freedom which couple directly to the quarks. At very high density the quarks and gluons become deconfined and the entire system is confined by a bag, or potential. For a list of references and recent work, see Ref. [2].

In a recent paper [3] the QMC model was generalized to include quark deconfinement at high density. Starting from a relativistic quark potential model [4], a change of Fock-space representation is implemented through a unitary transformation. The unitary operator is constructed in an extended Fock space in such a way that single compositehadrons of the model are redescribed in terms of elementaryparticle field operators. The unitary operator is constructed as a power series in the bound-state wave functions of the composite hadrons and application of the unitary operator on the original quark Hamiltonian leads to an unitarily equivalent Hamiltonian containing quark and explicit hadron degrees of freedom is constructed. The transformed Hamiltonian is composed of a sum of hermitian Hamiltonians endowed with a clear physical interpretation. In particular, one of such effective Hamiltonians describes the deconfinement of quarks. The new representation is known as the Fock-Tani representation [5]. In the present paper we use the lowest-order effective Hamiltonian to study properties of quark nuclear matter. Of particular interest are the equation of state (EOS) and transport equations for nucleons and deconfined quarks. We derive the Vlasov-Uehling-Ullenbeck (VUU) equations [6] for the distribution functions of nucle- 
ons and quarks in QNM. A numerical example is presented for the static EOS's for pure nuclear matter and QNM.

\section{The Model and the Effective Quark-Hadron Hamiltonian}

In the model of Ref. [3], a single-nucleon state in nuclear matter can be written in a second-quantized notation as (repeated indices are summed over)

$$
|\alpha\rangle=B_{\alpha}^{\dagger}|0\rangle, \quad B_{\alpha}^{\dagger}=\frac{1}{\sqrt{3 !}} \Psi_{\alpha}^{\mu_{1} \mu_{2} \mu_{3}} q_{\mu_{1}}^{\dagger} q_{\mu_{2}}^{\dagger} q_{\mu_{3}}^{\dagger},
$$

where the $q_{\mu}^{\dagger}$ 's are constituent-quark creation operators, $\Psi_{\alpha}^{\mu_{1} \mu_{2} \mu_{3}}$ is the Fock-space nucleon amplitude. The quark creation and annihilation operators satisfy canonical anticommutation relations. In Eq. (1), $\alpha$ denotes the spatial and internal quantum numbers of the nucleons, as internal and c.m. energies and the spin-isospin quantum numbers. The quark indices $\mu$ identify the spatial and internal quantum numbers as momentum, spin, flavor and color. The amplitude $\Psi_{\alpha}^{\mu_{1} \mu_{2} \mu_{3}}$ is taken to be orthonormalized.

The amplitude $\Psi_{\alpha}^{\mu_{1} \mu_{2} \mu_{3}}$ corresponds to a bound state of three constituent quarks, such that each constituent quark satisfies a Dirac equation of the form

$$
\left[-i \vec{\alpha} \cdot \vec{\nabla}+\beta^{0} m_{q}^{*}+1 / 2(1+\beta) V(r)\right] \psi(r)=E_{q}^{*} \psi(r),
$$

where $m_{q}^{*}=m_{q}-g_{\sigma}^{q} \sigma_{0}, E_{q}^{*}=\varepsilon^{*}-g_{\omega}^{q} \omega_{0}$.

In the situation that quarks remain confined to the interior of nucleons, the internal structure of the nucleon is manifest in the effective mass of the nucleon in medium [1]. In the mean field approximation, the energy density of symmetrical nuclear matter can be written as

$$
\frac{E}{V}=4 \int_{0}^{k_{F}} \frac{d^{3} p}{(2 \pi)^{3}} E_{N}^{*}(p)+\frac{1}{2} m_{\sigma}^{2} \sigma_{0}^{2}+\frac{1}{2} m_{\omega}^{2} \omega_{0}^{2},
$$

where $\rho_{B}=B / V$ is the baryon density and $m_{\sigma}$ and $m_{\omega}$ are the masses of $\sigma_{0}$ and $\omega_{0}$, and $E_{N}^{*}=\sqrt{p^{2}+M_{N}^{* 2}}$. The explicit form of $M^{*}$ is given in Ref [3]. The values of mean fields are determined as usual [1]: $\omega_{0}$ follows from its equation of motion and $\sigma_{0}$ is determined minimizing $E$ with respect to $\sigma_{0}$.

Now, the situation with deconfined quarks and composite hadrons simulataneously present in the system is very complicated, because the standard many-body techniques cannot be directly applied to the composite-hadron creation and annihilation operators $B_{\alpha}^{\dagger}$ and $B_{\alpha}$. This is because their anticommutation relations are noncanonical, namely

$$
\left\{B_{\alpha}, B_{\beta}^{\dagger}\right\}=\delta_{\alpha \beta}-\Delta_{\alpha \beta}, \quad\left\{B_{\alpha}, B_{\beta}\right\}=0,
$$

where

$$
\Delta_{\alpha \beta}=3 \Psi_{\alpha}^{* \mu_{1} \mu_{2} \mu_{3}} q_{\nu_{3}}^{\dagger}\left[\Psi_{\beta}^{\mu_{1} \mu_{2} \nu_{3}}-\frac{1}{2} \Psi_{\beta}^{\mu_{1} \nu_{2} \nu_{3}} q_{\nu_{2}}^{\dagger} q_{\mu_{2}}\right] q_{\mu_{3}} .
$$

In addition, one has

$$
\left\{q_{\mu}, B_{\alpha}^{\dagger}\right\}=\sqrt{\frac{3}{2}} \Psi_{\alpha}^{\mu \mu_{2} \mu_{3}} q_{\mu_{2}}^{\dagger} q_{\mu_{3}}^{\dagger}, \quad\left\{q_{\mu}, B_{\alpha}\right\}=0 .
$$

These equations reflect the internal structure of the hadrons, Eq. (6) in particular shows that the quark and nucleon field operators are not kinematically independent. It is precisely the noncanonical nature of these anticommutators that complicates the mathematical treatment of QNM.

One way to proceed is to change representation, such that composite field operators are redescribed in terms of elementary-particle field operators in an extended Fock space. This is done as follows. Initially, fictitious or ideal nucleons are introduced in close correspondence to the real single-nucleon states. Next, a unitary operator is constructed in the extended Fock space such that

$$
\left.\left.|\alpha\rangle=B_{\alpha}^{\dagger}|0\rangle \longrightarrow U^{-1}|\alpha\rangle \equiv \mid \alpha\right)=b_{\alpha}^{\dagger} \mid 0\right),
$$

where

$$
\left\{b_{\alpha}, b_{\beta}^{\dagger}\right\}=\delta_{\alpha \beta}, \quad\left\{b_{\alpha}, b_{\beta}\right\}=0,
$$

and $q \mid 0)=b \mid 0)=0$. In addition, in the new representation, one has $\left\{q_{\mu}, b_{\alpha}\right\}=\left\{q_{\mu}, b_{\alpha}^{\dagger}\right\}=0$. The unitary operator $U$ is constructed as a power series in the bound state amplitude $\Psi$. The meaning of this expansion can be understood as follwos. The term $\Delta_{\alpha \beta}$ plays no role and can be taken to be zero in situations that quarks remain confined in the interior of the nucleons, such as in systems at low densities. In this case there is no significant overlap of the internal structures of different nucleons and $U=1$. As the density of the system increases, quarks in different nucleons start to overlap and $\Delta_{\alpha \beta}$ cannot be neglected. The construction of $U$ as an expansion in powers of $\Psi$ 's takes into account the effects of the overlap of the internal quark structures of different nucleons.

When the unitary operator $U$ is applied to the microscopic quark Hamiltonian, one obtains [5] an effective Hamiltonian that describes all possible processes involving free quarks and composite nucleons. The quark Hamiltonian of Eq. (2) can be written as

$$
H_{q}=T_{q}+V_{q q}=T(\mu) q_{\mu}^{\dagger} q_{\mu}+\frac{1}{2} V_{q q}(\mu \nu ; \sigma \rho) q_{\mu}^{\dagger} q_{\nu}^{\dagger} q_{\rho} q_{\sigma},
$$

where $V_{q q}$ is the confining potential. Application of $U$ to $H_{q}$ leads to $H_{e f f}=U^{-1} H_{q} U$. The first nontrivial effects of the internal structure of the nucleons comes in with $U$ at first-order in $\Psi$,

$$
H_{e f f}^{(1)}=T_{q}+H_{b}+\tilde{V}_{q q}+V_{b q}+\cdots,
$$

where $\cdots$ indicate terms not relevant for our discussion here [5].

In Eq. (10), $H_{b}=T_{b}+V_{b b}$, where $T_{b}$ is a singlenucleon energy and $V_{b b}$ is an effective nucleon-nucleon interaction without quark exchange. $H_{b}$ leads to the normal 
QMC model, in which the many-body system is described by nonoverlapping nucleons - no quark-exchange. In particular, it describes Fock terms in the QMC model [7].

The term $\tilde{V}_{q q}$ contains two-quark and three-quark interactions. When $\Psi$ is a bound-state eigenstate of the original quark Hamiltonian, one obtains [5]

$$
V_{q q}=\frac{1}{2} V_{q q}(\mu \nu ; \sigma \rho) q_{\mu}^{\dagger} q_{\nu}^{\dagger} q_{\rho} q_{\sigma}-E_{\alpha} B_{\alpha}^{\dagger} B_{\alpha}
$$

This interaction leads to a quark Hamiltonian that has a positive semidefinite spectrum [5], i.e. the transformed Hamiltonian involving only quark operators describes only processes in the continuum. by

The term $V_{b q}$ is our main focus in this paper. It is given

$$
V_{b q}=V_{b q}\left(\mu_{1} \mu_{2} \mu_{3}, \beta\right) q_{\mu_{1}}^{\dagger} q_{\mu_{2}}^{\dagger} q_{\mu_{3}}^{\dagger} b_{\beta}+\text { h.c. },
$$

with

$$
\begin{aligned}
V_{b q}\left(\mu_{1} \mu_{2} \mu_{3}, \beta\right) & =\frac{1}{\sqrt{6}}\left[H_{q}\left(\mu_{1} \mu_{2} ; \sigma \rho\right) \Psi_{\beta}^{\sigma \rho \mu_{3}}\right. \\
& \left.-H_{q}(\mu \nu ; \sigma \rho) \Psi_{\beta}^{\sigma \rho \tau_{3}} \Delta\left(\mu_{1} \mu_{2} \mu_{3} ; \mu \nu \tau_{3}\right)\right]
\end{aligned}
$$

where $\Delta(\mu \nu \tau ; \sigma \rho \lambda)=\sum_{\alpha} \Psi_{\alpha}^{\mu \nu \tau} \Psi_{\alpha}^{* \sigma \rho \lambda}$. It can be shown [5] that when $\Psi$ is a stationary state of $H_{q}, V_{b q}=0$. The meaning of this is that the baryon bound state in stable against spontaneous decay in the absence of external perturbations, as in vacuum. The crucial observation [3] is that in a many-body system, the confining quark-quark interaction in general can become modified due to a variety of effects. Suppose we generate an effective quark-hadron interaction as in Eq. (12) using $\Psi$ 's that are eigenstates of the QMC Hamiltonian, but instead of the original confining interaction $V(r)$ we use a $V(r)$ that is deconfining. Then the term $V_{b q}$ is nonzero. This means that $V_{b q}$ will give rise to processes that lead to the decomposition of the nucleons into three quarks and vice-versa.

The interesting point now is to obtain the distribution functions for nucleons and quarks in the medium as a function of the total baryon density. The point is that at low densities, $V_{b q}=0$ and there will be only nucleons in the system. As the density increases, at some critical density the potential $V(r)$ starts to deconfine quarks and the systems becomes a mixture of nucleons and unconfined quarks. An important question to be answered is the following. Given at $t=0$ an initial quark-nucleon configuration and a density-dependent $V(r)$, what are the relative fractions of nucleons and quarks at time $t=T$ ? For sufficiently low densities, this question can be answered in the context of a VUU equation [6] for $n_{\alpha}$ and $n_{\mu}$.

\section{Vlasov-Uehling-Ullenbeck Equa- tions for Quark-Nuclear Matter}

We start defining the nucleon and quark density matrices as

$$
\rho_{b}(\beta, \alpha) \equiv\left\langle\Phi\left|b_{\alpha}^{\dagger} b_{\beta}\right| \Phi\right\rangle, \quad \rho_{q}(\nu, \mu) \equiv\left\langle\Phi\left|q_{\mu}^{\dagger} q_{\nu}\right| \Phi\right\rangle .
$$

The nucleon and quark creation and annihilation operators satisfy canonical anticommutation relations, since they are in the Fock-Tani representation. The time evolution of the nucleon density matrix $\rho_{b}(\beta, \alpha)$ is given by

$$
\begin{aligned}
i \frac{d}{d t} \rho_{b}(\beta, \alpha) & =\left[\rho_{b}(\beta, \alpha), H_{e f f}\right] \\
& =\left\langle\Phi\left|\left(\left[b_{\alpha}^{\dagger}, H_{e f f}\right] b_{\beta}+b_{\alpha}^{\dagger}\left[H_{e f f}, b_{\beta}\right]\right)\right| \Phi\right\rangle .
\end{aligned}
$$

A similar equation is obtained for the quark density matrix $\rho_{q}(\nu, \mu)$,

$$
\begin{aligned}
i \frac{d}{d t} \rho_{q}(\nu, \mu) & =\left[\rho_{q}(\nu, \mu), H_{e f f}\right] \\
& =\left\langle\Phi\left|\left(\left[q_{\mu}^{\dagger}, H_{e f f}\right] q_{\nu}+q_{\mu}^{\dagger}\left[H_{e f f}, q_{\nu}\right]\right)\right| \Phi\right\rangle .
\end{aligned}
$$

In order to evaluate the commutators in the equations above, one needs to know the structure of $H_{e f f}$ in terms of the $b$ and $q$ operators. The effective Hamiltonian $H_{e f f}$ in Eq. (10) contains two terms of the "usual" form, i.e. terms with a single-body kinetic plus two-body potential energies,

$$
\begin{aligned}
& H_{b}=E(\alpha) b_{\alpha}^{\dagger} b_{\alpha}+\frac{1}{2} V(\alpha \beta ; \delta \gamma) b_{\alpha}^{\dagger} b_{\beta}^{\dagger} b_{\gamma} b_{\delta}, \\
& \tilde{H}_{q}=T(\mu) q_{\mu}^{\dagger} q_{\mu}+\frac{1}{2} \tilde{V}_{q q}(\mu \nu ; \sigma \rho) q_{\mu}^{\dagger} q_{\nu}^{\dagger} q_{\rho} q_{\sigma},
\end{aligned}
$$

where $E(\alpha)=\left\langle\Psi_{\alpha}\left|H_{q}\right| \Psi_{\alpha}\right\rangle, \tilde{V}_{q q}$ describes quark-quark interactions in the continuum only and is unable to bind three quarks into nucleons [5]. $H_{\text {eff }}$ also contains a nondiagonal term of the form

$$
\tilde{V}_{b}=\sum_{\alpha \neq \beta} E(\alpha, \beta) b_{\alpha}^{\dagger} b_{\beta}
$$

where $E(\alpha, \beta)=\left\langle\Psi_{\alpha}\left|H_{q}\right| \Psi_{\beta}\right\rangle$ - in order to make clear the restriction $\alpha \neq \beta$ we have made use of the summation symbol. This term is obviously zero if $\Psi$ is an eigenstate of $H_{q}$, but in the present case that we use an unconfining $V(r)$ it is nonzero. The most interesting term in $H_{e f f}$ for us is $V_{b q}$ of Eq. (12). It leads to nucleon breakup and nucleon recombination.

The two terms in $H_{\text {eff }}$ of the "normal" form, Eqs. (17) and (18), lead to the usual VUU equations [6] and therefore will not be discussed at the moment. Since we are working in lowest order in $\Psi$, it is natural to consider a first-order perturbated wave function for the many-body system in the following form

$$
|\Phi\rangle=\left|\Phi_{0}\right\rangle+\left|\Phi_{b}\right\rangle+\left|\Phi_{b q}\right\rangle,
$$

where the unperturbated term $\left|\Phi_{0}\right\rangle$ is the product of timedependent Hartree-Fock (TDHF) state vectors of nucleons 
and quarks and the perturbated terms $\left|\Phi_{b}\right\rangle$ and $\left|\Phi_{b q}\right\rangle$ are given by

$$
\begin{aligned}
\left|\Phi_{b}\right\rangle & =\sum_{\alpha, \beta} \frac{1-e^{i \omega t}}{\omega} E(\alpha, \beta) b_{\alpha}^{\dagger} b_{\beta}\left|\Phi_{0}\right\rangle \\
\left|\Phi_{b q}\right\rangle & =\sum_{\alpha, \beta} \frac{1-e^{i \omega t}}{\omega}\left[V_{b q}\left(\mu_{1} \mu_{2} \mu_{3}, \alpha\right) q_{\mu_{1}}^{\dagger} q_{\mu_{2}}^{\dagger} q_{\mu_{3}}^{\dagger} b_{\alpha}\right. \\
& \left.+V_{b q}^{*}\left(\mu_{1} \mu_{2} \mu_{3}, \alpha\right) b_{\alpha}^{\dagger} q_{\mu_{3}} q_{\mu_{2}} q_{\mu_{1}}\right]\left|\Phi_{0}\right\rangle
\end{aligned}
$$

We make now the assumption of diagonal density matrices, which amounts to assuming

$$
\rho_{b}(\beta, \alpha)=\delta_{\alpha \beta} n_{b}(\alpha), \quad \rho_{q}(\nu, \mu)=\delta_{\mu \nu} n_{q}(\mu),
$$

where $n_{b}(\alpha)$ and $n_{q}(\mu)$ are the density distributions of nucleons and quarks, respectively. The contributions to the VUU equations from the different components of $|\Phi\rangle$ in Eq. (20) for the time evolution of $n_{b}(\alpha)$ and $n_{q}(\mu)$ can be written a sum of contributions of the separate components, since there is no mixing of the different components of $|\Phi\rangle$ in the expectation values of Eqs. (15) and (16).

The component $\left|\Phi_{0}\right\rangle$ of $|\Phi\rangle$ leads to the usual TDHF contribution and will not be discussed at the moment. The evaluation of the contribution of $\left|\Phi_{b}\right\rangle$ is relatively easy. It can be written as

$$
\begin{aligned}
& \left.\frac{d}{d t} n_{b}(\alpha)\right|_{\left|\Phi_{b}\right\rangle}=2 \pi \sum_{\beta \neq \alpha}|E(\alpha, \beta)|^{2} \delta\left(E_{\alpha}-E_{\beta}\right) \\
& \times\left\{n_{b}(\beta)\left[1-n_{b}(\alpha)\right]-n_{b}(\alpha)\left[1-n_{b}(\beta)\right]\right\} \cdot
\end{aligned}
$$

The term in curly brackets represents the effect of the Pauli exclusion principle and can easily be interpreted: the distribution function $n(\alpha)$ will be changed positively (first term) by filling unoccupied states $\beta$, or it will be diminished by removing occupied states $\beta$ (second term).

The derivation of the contribution of the component $\left|\Phi_{b q}\right\rangle$ of $|\Phi\rangle$ is much more complicated. After a rather lengthly calculation, the contribution for the equation for $n_{b}(\alpha)$ can be written as

$$
\begin{aligned}
& \left.\frac{d}{d t} n_{b}(\alpha)\right|_{\left|\Phi_{b q}\right\rangle}=2 \pi \sum_{\mu^{\prime} \nu^{\prime} \sigma^{\prime}} 6\left|V_{b q}\left(\mu^{\prime} \nu^{\prime} \sigma^{\prime}, \alpha\right)\right|^{2} \delta\left(E_{\alpha}-E_{q^{\prime} s}\right)\left\{\left[1-n_{b}(\alpha)\right] n_{q}\left(\mu^{\prime}\right) n_{q}\left(\nu^{\prime}\right) n_{q}\left(\sigma^{\prime}\right)\right. \\
& \left.-n_{b}(\alpha)\left[1-n_{q}\left(\mu^{\prime}\right)+3 n_{q}\left(\mu^{\prime}\right) n_{q}\left(\nu^{\prime}\right)-n_{q}\left(\mu^{\prime}\right) n_{q}\left(\nu^{\prime}\right) n_{q}\left(\sigma^{\prime}\right)\right]\right\},
\end{aligned}
$$

where $E_{q^{\prime} s}=E_{\mu^{\prime}}+E_{\nu^{\prime}}+E_{\sigma^{\prime}}$. And the contribution for the equation for $n_{q}(\mu)$ can be written as

$$
\begin{aligned}
& \left.\frac{d}{d t} n_{q}(\mu)\right|_{\left|\Phi_{b q}\right\rangle}=2 \pi \sum_{\alpha^{\prime} \nu^{\prime} \sigma^{\prime}} 18\left|V_{b q}\left(\mu^{\prime} \nu^{\prime} \sigma^{\prime}, \alpha^{\prime}\right)\right|^{2} \delta\left(E_{q^{\prime} s}-E_{\alpha^{\prime}}\right) \\
& \times\left\{\left[1-n_{q}\left(\sigma^{\prime}\right)+n_{q}\left(\nu^{\prime}\right) n_{q}\left(\sigma^{\prime}\right)+2 n_{q}(\mu) n_{q}\left(\nu^{\prime}\right)-n_{q}(\nu) n_{q}\left(\nu^{\prime}\right) n_{q}\left(\sigma^{\prime}\right)\right] n_{b}\left(\alpha^{\prime}\right)\right. \\
& \left.-n_{q}(\mu) n_{q}\left(\nu^{\prime}\right) n_{q}\left(\sigma^{\prime}\right)\left[1-n_{b}\left(\alpha^{\prime}\right)\right]\right\}
\end{aligned}
$$

where now $E_{q^{\prime} s}=E_{\mu}+E_{\nu^{\prime}}+E_{\sigma^{\prime}}$.

The numerical solution of the VUU equations is not simple, and explicit solutions will be presented elsewhere. The techniques used in Ref. [8] might be particularly useful in the present context. In order to have a feeling of what can be expected for an explicit solution, in the following section a static EOS for QNM is obtained.

\section{Equation of State}

One expects that for a given baryon density $\rho_{B}=B / V$, where $B$ is total baryon number, the equilibrium solution of the VUU equations discussed in the previous section give rise to two Fermi seas, one for baryons and one for quarks. The two Fermi seas satisfy the constraint

$$
\sum_{\alpha} n_{b}(\alpha)=Z B, \quad \sum_{\mu} n_{q}(\mu)=(1-Z) B,
$$

where $Z$ measures the fraction of nucleons in the medium. Of course, the problem is to estimate the value of $Z$.

Here we follow Ref. [3], but instead of the linearly rising confining potential used there, we use an harmonic oscillator,

$$
V(r)=\frac{1}{2} k r^{2} e^{-\mu^{2} r^{2}}
$$

where $\mu$ is a function of $\rho_{B}$ such that it is zero for $\rho_{B} \leq 3 \rho_{0}$, where $\rho_{0}$ is the saturation density of normal nuclear matter, and for $\rho_{B}>3 \rho_{0}$ it is given by $\mu=\rho_{B} / 3 \rho_{0}-1$. The aim of changing $V(r)$ is to investigate the sensitivity of the results to the confining potential. The energy density of the system 
can be written as

$$
\begin{aligned}
\frac{E}{V} & =4 \int_{0}^{k_{F}^{b}} \frac{d^{3} p}{(2 \pi)^{3}} E_{N}^{*}(p)+\frac{1}{2} m_{\sigma}^{2} \sigma_{0}^{2} \frac{1}{2} m_{\omega}^{2} \omega_{0}^{2} \\
& +12 \int_{0}^{k_{F}^{q}} \frac{d^{3} k}{(2 \pi)^{3}} E_{q}^{*}(k),
\end{aligned}
$$

where the Fermi momenta $k_{F}^{b}$ and $k_{F}^{q}$ are given as

$$
\rho_{b}=2\left(k_{F}^{b}\right)^{2} / 3 \pi^{2}, \quad \rho_{q}=2\left(k_{F}^{q}\right)^{2} / \pi^{2},
$$

with $\rho_{b}=Z \rho_{B}$ and $\rho_{q}=(1-Z) \rho_{B}$, and $E_{q}^{*}(k)=$ $\sqrt{k^{2}+m_{q}^{* 2}}$.

Of course, the hard problem is to calculate the factor $Z$. Here we use a perturbative approach to evaluate $Z$, following Ref. [3]. We use $m_{q}=313 \mathrm{MeV}$ and $k=1 \mathrm{GeV}$. The coupling constants in the present case are found to be $g_{\sigma}^{q}=6.34$ and $3 g_{\omega}^{q}=5.67$, and the incompressibility is $K=253 \mathrm{MeV}$. The EOS's for nuclear matter and QNM are shown in Fig. 1. As in Ref. [3], one sees that the present EOS of QNM is softer then the one of pure nuclear matter, and presents small differences with the latter for densities close to the deconfining density.

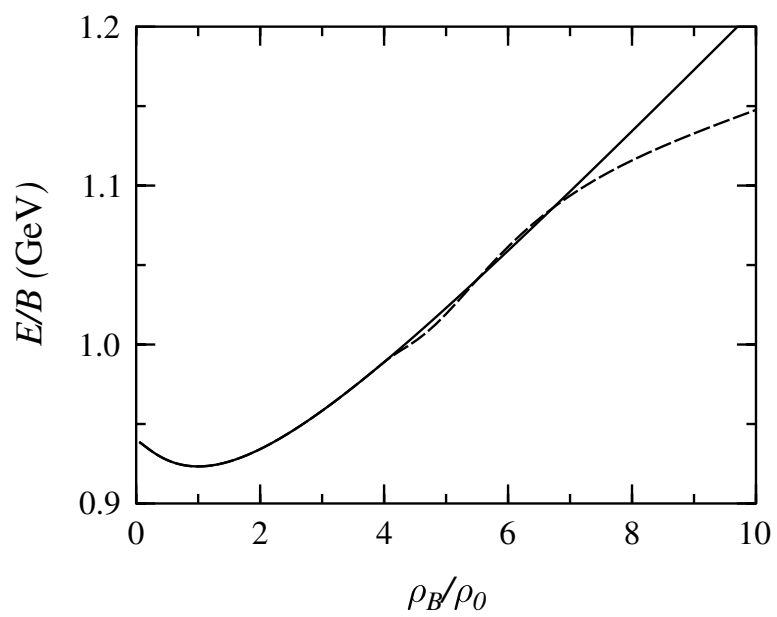

Figure 1. The solid line is the EOS of pure nuclear matter and the dashed line is the EOS of QNM.

\section{Conclusions}

A model was introduced to study quark deconfinement in hadronic matter. The model is based on a relativistic microscopic quark Hamiltonian with a density dependent quarkquark interaction. Nucleon and deconfined quark degrees of freedom are treated on equal footing using a mapping procedure. Transport equations of the VUU-type were obtained and the static EOS for pure nuclear matter and QNM were calculated. Future work includes the numerical solution of the VUU equations and the study of implications of our EOS for the phenomenology of compact stars.

\section{Acknowledgments}

The work of GK was partially supported by FAPESP and CNPq.

\section{References}

[1] P. A. M. Guichon, Phys. Lett. B200, 235 (1988); K. Saito and A.W. Thomas, Phys. Lett. B327, 9 (1994).

[2] P.K. Panda, M.E. Bracco, E. Conte, and G. Krein, Phys. Rev. C 65, 065206 (2002)

[3] G. Krein and V.E. Vizcarra, arXiv:nucl-th/0206047.

[4] H. Toki, U. Meyer, A. Faessler, and R. Brockmann, Phys. Rev. C 58, 3749 (1998).

[5] D. Hadjimichef, G. Krein, S. Szpigel, and J.S. da Veiga, Phys. Lett. B367, 317 (1996); Ann. Phys. (NY) 268, 105 (1998).

[6] H. Stoecker and W. Greiner, Phys. Rep. 137, 277 (1986).

[7] G. Krein, A.W. Thomas, and K. Tsushima, Nucl. Phys. A650, 313 (1999).

[8] G.A. Gonçalves, G. Orengo, M.T. Vilhena, and C.O. da Graça, Ann. Nucl. Energy 29, 561 (2002). 